\title{
Small intestinal mucosal patterns of coeliac disease and idiopathic steatorrhoea seen in other situations
}

\author{
R. R. W. TOWNLEY, M. H. CASS, AND CHARLOTTE M. ANDERSON \\ From the Gastroenterological Research Unit, Royal Children's Hospital Research Foundation, \\ Royal Children's Hospital, Melbourne, Australia
}

EDITORIAL SYNOPSIS This paper suggests that the flat type of intestinal mucosa seen in coeliac disease is a property of response inherent in the mucosa and not specific to the damaging agent.

In idiopathic steatorrhoea of adults and coeliac disease of children, the appearance of the proximal small intestinal mucosa is abnormal, the main features being absence of the normal villous pattern, flattening and irregularity of the surface epithelial cells, and infiltration of the lamina propria with plasma cells. Paulley (1954), Sakula and Shiner (1957), and Himes and Adlersberg (1958) were some of the early workers to demonstrate these changes, which have been amply confirmed by others. However, the specificity of the changes has not been so clearly defined. Rubin, Brandborg, Phelps, and Taylor (1960) discussed a number of reports of disturbances of small intestinal mucosal morphology in clinical and experimental situations and concluded that, with the exception of tropical sprue, the 'flat' mucosal morphology typically found in idiopathic steatorrhoea and coeliac disease is specific to these disorders. Cameron, Astley, Hallowell, Rawson, Miller, French, and Hubble (1962) found their experience in children in accord with this, although they felt that lesser degrees of mucosal abnormality could be seen in other conditions, e.g. Giardia lamblia infestation. Fone, Meynell, Harris, Cooke, Brewer, and Cox (1960) divided their jejunal biopsies from adult patients with steatorrhoea into two groups, on the basis of whether the mucosa appeared 'flat', or had an 'abnormal villous' pattern, descriptive terms which we have adopted in the present paper. Fone et al. felt that the difference in appearance between their two groups reflected the operation of different aetiological factors.

The present paper reports observations on small intestinal mucosal lesions, similar to those of coeliac disease, produced experimentally in animals and observed under certain other conditions in children, and advances a hypothesis for the development of these lesions.

\section{MATERIALS AND METHODS}

ANIMAL MATERIAL In the first experiment a $30 \mathrm{~cm}$. segment of jejunum of an adult mongrel dog was isolated. Continuity of the remainder of the bowel was restored by anastomosis. The isolated jejunal segment, attached to its mesentric pedicle, was placed transversely under the skin of the abdominal wall and the ends of the segment were brought out to open on each side of the abdominal wall.

Three months later the wounds were well healed, and the ends of the jejunal segment appeared healthy. At this time intermittent instillation of normal hydrochloric acid into the proximal stoma of the segment was commenced. Six ml. of N HCL was instilled daily for 10 days, then every second day for six days and then twice weekly for two and a half weeks.

Biopsies of the mucosa about half way along the isolated segment were obtained by the multipurpose suction biopsy tube of Brandborg, Rubin, and Quinton (1959) before, and at various times after, instillation of acid was begun.

Ten weeks after acid instillation was stopped, the isolated segment was removed, and sections of the segment were examined histologically.

Second experiment A jejunal segment was isolated from the bowel of a second dog, by the method of the previous experiment, but in this case the segment was exteriorized and opened along its antimesenteric border. The cut edges of the segment were sewn to the skin of the abdominal wall to keep the mucosa exposed.

HUMAN MATERIAL Biopsies were obtained from (1) the stump of an ileostomy one week after its formation in a 1-day-old infant with meconium ileus due to fibrocystic disease of the pancreas; (2) an ileal conduit which had been fashioned five years previously in a boy with ectopia vesicae.

All biopsy specimens of human and animal origin were placed on a piece of filter paper with the epithelial surface uppermost, fixed in formalin, and prepared for histological examination in the usual way. The sections were stained with haematoxylin and eosin. 

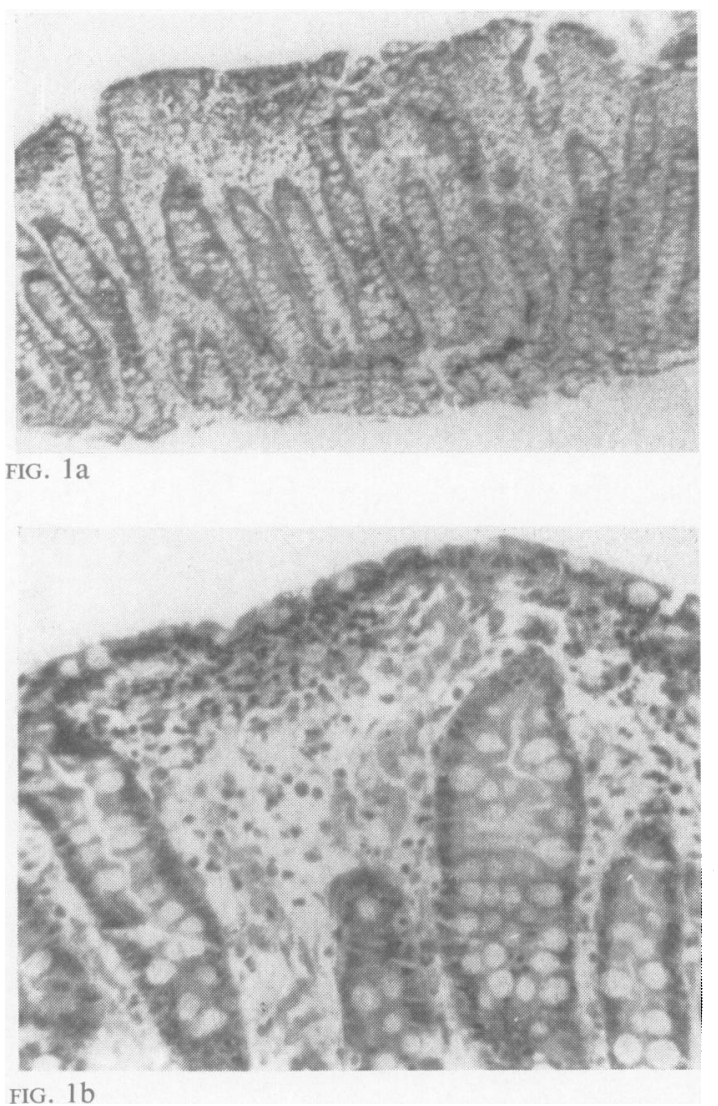

FIG. 1. Biopsy from mucosa of isolated loop of dog's jejunum after intermittent instillation of hydrochloric acid for eight weeks. (a) Generalized flat appearance. Haematoxylin and eosin $\times 63 \cdot 5$. (b) Flattening and irregularity of surface epithelium, and plasma cell infiltration of lamina propria. Haematoxylin and eosin $\times 180$

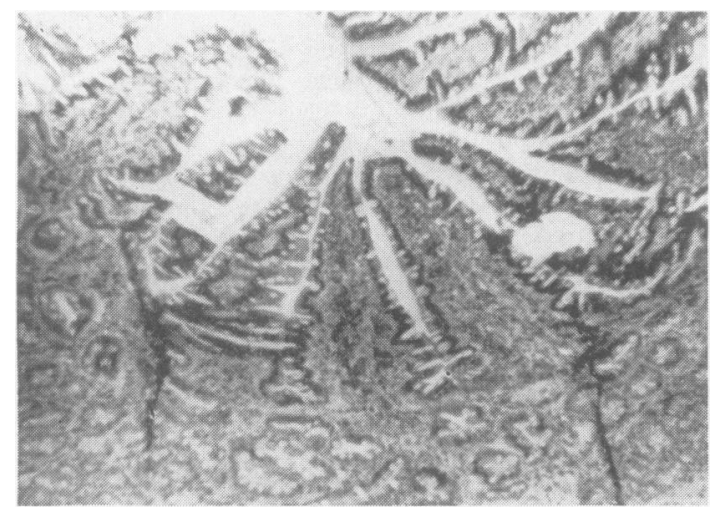

FIG. 2. Section of dog's jejunal loop 10 weeks after acid instillation had been stopped. Reconstitution of a villous pattern has occurred. Haematoxylin and eosin $\times 62.5$
RESULTS

IN ANIMAL EXPERIMENTS Sections of the biopsy obtained from the first dog before acid instillation was started showed normal villi covered with columnar epithelium. Specimens obtained during the first few weeks of acid instillation showed some ulceration and infiltration of the lamina propria with polymorphonuclear leucocytes. However, by eight weeks after commencing intermittent instillation of acid the mucosa had assumed a 'flat' appearance remarkably similar to that seen in idiopathic steatorrhoea, with irregularity and flattening of the surface epithelium and plasma cell infiltration of the lamina propria (Fig. 1).

Mucosal specimens obtained after instillation of acid had been stopped showed a gradual return to a normal, or near normal, pattern. The epithelial cells regained a normal appearance within five weeks but reconstitution of a villous pattern was much more delayed. Sections of the jejunal segment when it was removed at the conclusion of the experiment 10 weeks after stopping acid instillation showed a wellmarked villous pattern, although by this time the segment was considerably narrowed (Fig. 2).

Essentially similar morphological changes were produced by incidental recurrent trauma and licking of the exposed piece of bowel mucosa in the second dog.

IN HUMAN MATERIAL Sections from the most exposed portion of the ileostomy stump of the first patient showed a flat appearance (Fig. 3a) but sections from a more protected region nearby had a more normal pattern (Fig. 3b). There was a gradual transition between these areas, where the villi were thickened, and covered with flattened epithelium.

Sections from the ileal conduit of the second patient showed an overall flat appearance (Fig. 4a). although the surface epithelium was cuboidal, and there were increased numbers of goblet cells (Fig. 4b).

\section{DISCUSSION}

The observations recorded in this paper demonstrate that a variety of types of chronic damage to the small intestinal mucosa may lead to a loss of the normal villous pattern, resulting in a flat appearance similar to the most severe lesions encountered in idiopathic steatorrhoea and coeliac disease. Thus, this altered morphology would appear to reflect a property of response inherent in the mucosa, and not specific to the damaging agent.

In our clinical experience, which is confined to white children in a temperate climate, the really flat mucosal lesion has not been encountered except in 


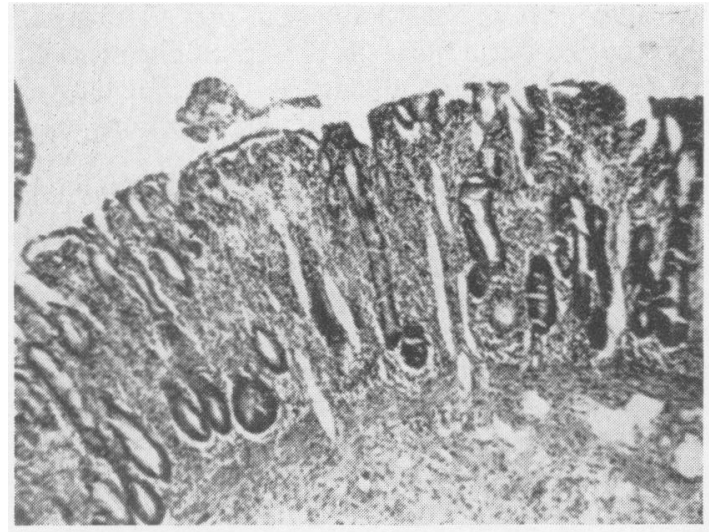

FIG. 3a

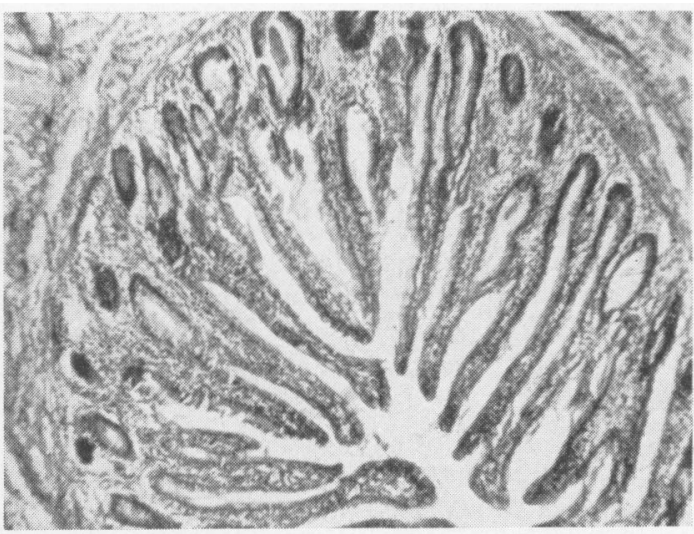

FIG, $3 \mathrm{~b}$

FIG. 3. Biopsies from the ileostomy of first patient. (a) From exposed mucosa, showing flat pattern. Haematoxylin and eosin $\times 62.5$. (b) From adjacent, more protected mucosa showing well-marked villous pattern. Haematoxylin and eosin $\times 62.5$

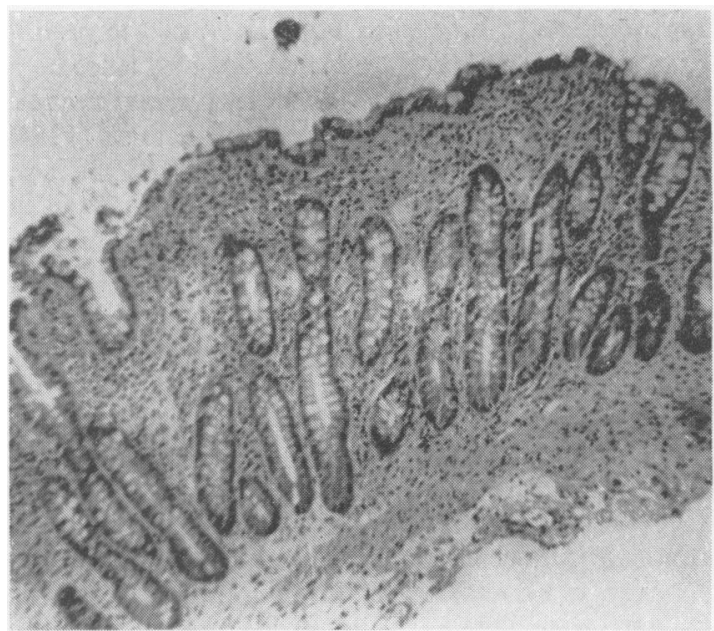

FIG. $4 \mathrm{a}$

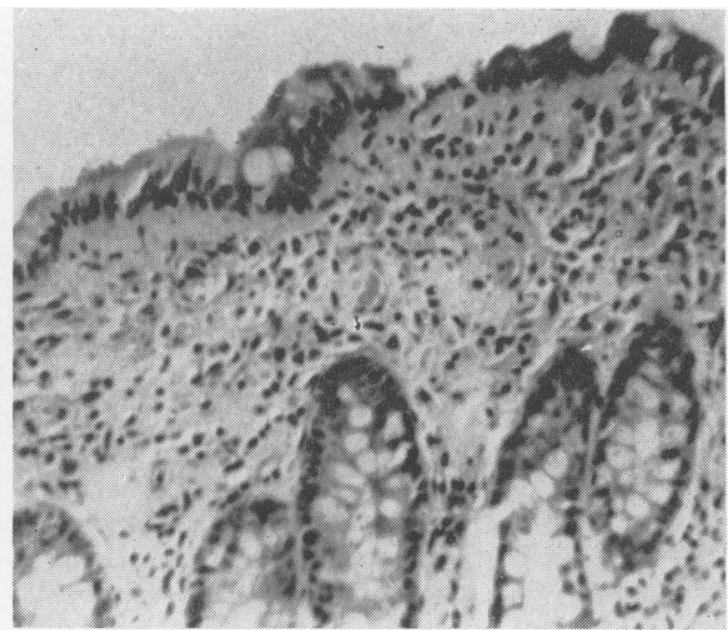

FIG. $4 \mathrm{~b}$

FIG. 4. Biopsy obtained from interior of an ileal conduit in second patient. (a) Showing overall flat pattern. Haematoxylin and eosin $\times 62.5$. (b) Showing fairly normal surface epithelium with increased numbers of goblet cells. The lamina propria shows infiltration by plasma cells. Haematoxylin and eosin $\times 180$

cases of coeliac disease subsequently responding to a gluten-free diet, and in the patients discussed in this paper, where the aetiological agent was apparent, in that the mucosa has obviously been exposed to a different environment. Therefore. there has been no diagnostic confusion when a flat mucosal pattern has been found.

However, it is evident from the literature that the situation is not so clear-cut in adult patients. For instance, some adult patients with steatorrhoea and a flat mucosal appearance do not seem to respond to an adequate gluten-free diet. It is therefore possible that there may be other causes of the mucosal changes in these patients. Certainly, although an abnormal villous pattern is more usual, a flat mucosal lesion may be found at times in patients with tropical sprue, a disorder widely believed to be different from idiopathic steatorrhoea (Butterworth and Perez-Santiago, 1958; Doniach and Shiner, 1957).

This mention of abnormal villous pattern brings one to a consideration of the significance of lesser degrees of disturbance of morphology in small intestinal mucosal biopsies. Abnormalities of mucosal pattern of insufficient severity to be described as flat are not infrequently met. The villi 
in such cases have been described as 'oedematous', 'swollen', 'clubbed', or 'irregular', whilst there are often also minor abnormalities of the surface epithelium and increased infiltration of the lamina propria, although in our experience this last feature is often difficult to assess.

Doniach and Shiner (1957) reported an abnormal villous pattern in duodenal biopsies from a patient with gastric ulcer, a patient with duodenal ulcer, and a patient with iron-deficiency anaemia. Baird and Dodge (1957) described changes in jejunal biopsies obtained from post-gastrectomy patients. Yesner, Schwartz, and Spiro (1960) reported abnormalities in duodenal biopsies from some patients with 'severe diarrhoea' and in the jejunal biopsy of a post-gastrectomy patient. Rubin et al. (1960) felt that an 'abnormal villous' appearance could be found in coeliac disease, but seemed hesitant to accept as abnormal the mucosal histology found by Baird and Dodge (1957) in post-gastrectomy patients, and by Jacobson, Prior, and Faloon (1960) in patients with steatorrhoea associated with administration of neomycin.

Cameron et al. (1962) demonstrated minor changes in the upper intestinal mucosa of some children with giardiasis or with iron-deficiency anaemia. We also have encountered minor abnormalities of duodenal mucosal pattern in some children with giardiasis or with iron-deficiency anaemia, and also in two children with cirrhosis of the liver (unpublished observations).

All the untreated patients of Cameron et al. (1962) with coeliac disease had severe mucosal lesions, although there was some variation in the degree of severity. However, we have shown (Anderson and Townley, 1962) in a group of 37 children diagnosed as suffering from coeliac disease and subsequently responding to a gluten-free diet, that there was considerable variation in the severity of change present in the biopsies obtained before treatment. A range of appearances from completely flat to an abnormal villous appearance was found within the group, although the former type of appearance was found in the majority.

Cameron et al. (1962) confirmed the observation of Anderson (1960) that the mucosal changes of coeliac disease usually revert to or toward normal during treatment with a gluten-free diet, and suggested that such improvement 'reflects in reverse the evolution of the characteristic coeliac change'. Our findings (Anderson and Townley, 1962) support this concept.

It is therefore evident that minor mucosal abnormality may exist in coeliac disease and idiopathic steatorrhoea, and also in a variety of other situations both in adults and children.
Evidence also points to the fact that these minor changes are but a stage in the development of the flat pattern, and strengthens the view that they are non-specific, reflecting a mucosal reaction to an abnormal environment.

It is recognized that the duodenal mucosal pattern of normal subjects is more irregular than that of normal jejunal mucosa. This is particularly evident when biopsy specimens are examined by the dissecting microscope. Duodenal mucosa is more exposed to minor degrees of damage by hydrochloric acid and food fragments than is the more distal small gut, and the jejunal mucosa in postgastrectomy is similar in this regard. Possibly such 'normal' damage may contribute to the irregularity of mucosal appearance in these sites.

So far, no explanation of the development of the abnormal mucosal morphology of idiopathic steatorrhoea has been universally accepted. In fact, remarkably divergent views on this subject have appeared, and no detailed hypothesis of the pathogenesis of the mucosal lesion has been advanced.

Paulley (1954) regarded the lesion as chronic inflammatory in type, Doniach and Shiner (1957) have used the term 'villous atrophy', Himes and Adlersberg (1958) refer to 'villous fusion', whilst Rubin et al. (1960) suggested abnormalities in production and multiplication of epithelial cells as the basis for the abnormal morphology, a view to which Padykula, Strauss, Ladman, and Gardner (1961) lend some support.

The results reported in the present paper show that a flat mucosal lesion may result from chronic damage of various types, and correlation of the clinical findings of ourselves and others suggests that minor degrees of change are related to the flat lesion but are also non-specific. All types of damage may be expected to evoke an inflammatory response, and we agree with Paulley (1959) that chronic inflammation is perhaps the basis of the mucosal alteration in coeliac disease. However, we feel that the inflammation is of 'chemical' rather than infective origin, due to an irritation by a product of gluten digestion, and that the presence of bacteria in some cases, as reported by Paulley (1959), is probably secondary rather than primary.

If a damaging agent, as we believe gluten to be in the patient with coeliac disease, is present in the intestinal lumen, it is likely that it will injure most severely the most exposed and differentiated tissue. We would suggest that such injury is the basis of the changes in the surface cells described by Padykula et al. (1961) as representing 'rapid dedifferentiation or modulation'. If damage to exposed cells be sufficiently severe, it is easy to imagine that denudation of epithelium and its basement membrane 
may result, and when neighbouring villi are involved, conditions are ripe for adherence of their raw surfaces.

If damage is recurrent or chronic, progressive fusion can be envisaged, where clumps of fused villi fuse again with each other, producing eventually a flat pattern. Observations on flat mucosal specimens are in accord with this concept, in that the total mucosal thickness is usually little different from normal and there are many more glands cut through in the deeper layers of the mucosa than there are openings on the surface. Many interconnexions between the deeper glands can be traced on serial sections, and one would expect to see considerable glandular dilatation if no drainage of secretion to the surface were possible. We feel that these findings are better explained as the result of fusion of overlying villi rather than stemming from glandular hypertrophy and proliferation as suggested by Doniach and Shiner (1957).

Increased numbers of mitotic figures in the crypts are observed in the flat mucosal biopsies (Shiner and Doniach, 1960), an expected mechanism if excess losses of surface epithelium are to be compensated.

The process of villous fusion has the advantage of protecting the deeper parts of the mucosa, although the disadvantage of diminished absorptive area is an inevitable accompaniment.

The mechanism of improvement of mucosal appearance during treatment of patients with coeliac disease is more difficult to conceive, but it is suggested that epithelial 'burrowing', a capacity of epithelium well recognized in other pathological and embryological situations, is responsible, and that the operation of factors which maintain normal morphology in normal subjects is allowed to resume in coeliac patients when gluten is removed from the diet.

\section{CONCLUSIONS}

The flat intestinal mucosal morphology seen in idiopathic steatorrhoea and coeliac disease has been observed both in experimental situations in animals and surgically produced situations in children where recurrent damage of various types has been operating, indicating that the altered morphology is a property of response inherent in the mucosa and not specific to the damaging agent.

In clinical paediatric practice the flat intestinal appearance is probably confined to coeliac disease although theoretically not therefore pathognomonic of it.
Lesser degrees of mucosal disturbance and abnormality in villous pattern represent a stage in the development of the flat pattern but are clinically more difficult to interpret. They occur in coeliac disease but are also seen in other disorders such as iron-deficiency anaemia or giardiasis.

An hypothesis of the development of the flat mucosal appearance is advanced in which it is envisaged that by a process of progressive villous fusion, successive degrees of abnormality of mucosal morphology are produced, culminating in the flat lesion. All degrees of mucosal abnormality may be encountered in this continuous process, the appearance at the time of biopsy reflecting the duration of action and the severity of the damaging agent.

REFERENCES

Anderson, C. M. (1960). Histological changes in the duodenal mucosa in coeliac disease. Arch. Dis. Childh., 35, 419-427.

- , and Townley, R. R. W. (1962). The effects of a gluten-free diet on intestinal histology in coeliac disease. In Intestinal Biopsy, Ciba Foundation Study Group No. 14, p. 39. Churchill, London.

Baird, I. M., and Dodge, O. G. (1957). Jejunal biopsy after partial gastrectomy. Quart. J. Med., 26, 393-400.

Brandborg, L. L., Rubin, G. E., and Quinton, W. E. (1959). A multipurpose instrument for suction biopsy of the oesophagus, stomach, small bowel and colon. Gastroenterology, 37, 1-16.

Butterworth, C. E., Jr., and Perez-Santiago, E. (1958). Jejunal biopsies in sprue. Ann. intern. Med., 48, 8-29.

Cameron, A. H., Astley, R., Hallowell, M., Rawson, A. B., Miller, C. G., French, J. M., and Hubble, D. V. (1962). Duodenojejunal biopsy in the investigation of children with coeliac disease. Quart. J. Med., 31, 125-140.

Doniach, I. and Shiner, M. (1957). Duodenal and jejunal biopsies. II. Histology. Gastroenterology, 33, 71-86.

Fone, D. J., Meynell, M. J., Harris, E. L., Cooke, W. T., Brewer, D. B., and Cox, E. V. (1960). Jejunal biopsy in adult coeliac disease and allied disorders. Lancet, 1, 933-938.

Himes, H. W., and Adlersberg, D. (1958). Pathologic changes in the small bowel in idiopathic sprue: biopsy and autopsy findings. Gastroenterology, 35, 142-154.

Jacobson, E. D., Prior, J. T., and Faloon, W. W. (1960). Malabsorptive syndrome induced by neomycin: morphologic alterations in the jejunal mucosa. J. Lab. clin. Med., 56, 245-250.

Padykula, H. A., Strauss, E. W., Ladman, A. J., and Gardner, F. H. (1961). A morphologic and histochemical analysis of the human jejunal epithelium in nontropical sprue. Gastroenterology, 40, 735-765.

Paulley, J. W. (1954). Observations on the aetiology of idiopathic steatorrhoea; jejunal and lymph-node biopsies. Brit. med. J., 2, 1318:1321.

- (1959). Jejunal mucosa in idiopathic steatorrhoea. Lancet, 2, 646-648.

Rubin, C. E., Brandborg, L. L., Phelps, P. C., and Taylor, H. C., Jr., (1960). Studies of celiac disease. 1. The apparent identical and specific nature of the duodenal and proximal jejunal lesion in celiac disease and idiopathic sprue. Gastroenterology, 38, 28-49.

Sakula, J., and Shiner, M. (1957). Coeliac disease with atrophy of the small-intestine mucosa. Lancet, 2, 876-877.

Shiner, M., and Doniach, I. (1960). Histopathologic studies in steatorrhea. Gastroenterology, 38, 419-440.

Yesner, R., Schwartz, R. D., and Spiro, H. M. (1960). Duodenal biopsy in diarrhea and steatorrhea. Yale J. Biol. Med., 32 , 361-369. 